13

\title{
Влияние контактной разности потенциалов на вольт-амперные характеристики в сканирующей туннельной спектроскопии
}

\author{
(C) М.В. Кузьмин, М.А. Митцев \\ Физико-технический институт им. А.Ф. Иофрфе РАН, \\ 194021 Санкт-Петербург, Россия \\ e-mail: m.kuzmin@mail.ioffe.ru
}

Поступило в Редакцию 1 апреля 2021 г.

В окончательной редакции 13 июня 2021 г.

Принято к публикации 16 июня 2021 г.

С помощью энергетических диаграмм асимметричных потенциальных барьеров, которые формируются при контакте двух металлов, обладающих различными работами выхода, рассмотрено влияние контактной разности потенциалов на вольт-амперные характеристики и спектры дифференциальной проводимости, получаемые с помощью сканирующей туннельной спектроскопии. Показано, что полученные выводы качественно согласуются с экспериментальными результатами для нанопленок иттербия толщиной 16 монослоев $(6.08 \mathrm{~nm})$. Однако количественно они существенно различаются. Проведен анализ этих различий.

Ключевые слова: поверхность, электронная структура, контактная разность потенциалов, энергетическая диаграмма, потенциальный барьер, сканирующая туннельная спектроскопия.

DOI: $10.21883 /$ JTF.2021.11.51542.91-21

\section{Введение}

Известно, что метод сканирующей туннельной спектроскопии (СТС) дает возможность получать важную информацию о поверхности и приповерхностных слоях исследуемых объектов. К настоящему моменту возможности метода рассмотрены в целом ряде монографий, обзорах и статьях [1-4]. В них отмечено, в частности, что метод позволяет исследовать плотность заполненных и незаполненных состояний. Показано также как такие исследования могут быть проведены. Для этого необходимо в экспериментах регистрировать зависимости туннельного тока $I$ с острия в исследуемый объект или в обратном направлении от разности потенциалов $V$, приложенной между объектом и острием (напряжения смещения), а также аналогичные зависимости для дифференциальной проводимости $d I / d V$ и нормированной дифференциальной проводимости $d I / d V /(I / V)$. Величина напряжения смещения в указанных экспериментах не превышает, как правило, 2 V. Значение контактной разности потенциалов (КРП) между исследуемым объектом и острием может достигать значений, превышающих $1 \mathrm{~V}$, т. е., по порядку величины оно может быть сопоставимо с величиной напряжений смещения, используемых при проведении экспериментальных исследований. Из сказанного следует, что должен быть проведен детальный анализ возможного влияния КРП на получаемые с помощью СТС результаты. В более ранних исследованиях $[5,6]$ подобный анализ проводился для модельной системы, которая включала в себя два металлических электрода (Al и $\mathrm{Au}$ ) с различными работами выхода, разделенные тонким слоем диэлектрика (пленкой $\mathrm{Al}_{2} \mathrm{O}_{3}$ толщиной $\leq 100 \AA$ ). В этих исследованиях одним из важных отличий от случая СТС является то, что поверх- ности обоих электродов плоские (т.е., имеют бесконечный радиус кривизны) и, следовательно, с их помощью нельзя моделировать процессы, происходящие вблизи поверхности острия. Поэтому до настоящего времени вопрос о влиянии КРП на результаты СТС остается открытым. В настоящей работе сделана попытка хотя бы частично восполнить существующий пробел.

\section{1. Модель, используемая для анализа влияния КРП на исследования с помощью СТС}

Рассматривается простейшая модель контакта двух металлов: исследуемый объект 1 и острие 2. Однако, несмотря на простоту, она позволяет выяснить особенности влияния КРП на результаты, получаемые с помощью СТС. На рис. 1, $a$ представлена ситуация, когда 1 и 2 находятся на большом друг от друга расстоянии, т.е. когда между ними нет обмена электронами. Если теперь металлы сблизить на малое расстояние или соединить проводником (при этом смещение $V$ равно нулю), то между ними произойдет обмен электронами. В результате перетекания электронов уровни Ферми сравняются, а между 1 и 2 установится контактная разность потенциалов, равная $V_{2.1}=\left(\varphi_{2}-\varphi_{1}\right) / e$, где $\varphi_{1}$ и $\varphi_{2}-$ работы выхода объекта и острия соответственно, $e-$ заряд электрона (рис. $1, b$ ). Если теперь прикладывать между 1 и 2 разность потенциалов любого знака (напряжение смещения $V \neq 0$ ), то можно регистрировать зависимости тока I между объектом и острием (вольт-амперные характеристики $(\mathrm{BAX}))$, а также зависимости $d I / d V$ от напряжения смещения. При $V<0$ (рис. 1,c) указанные зависимости будут содержать в себе информацию 

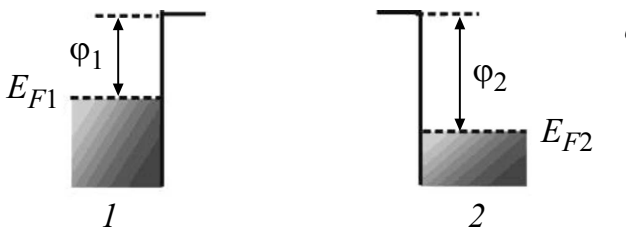

\section{$a$}

$V_{1,2}=\left(\varphi_{2}-\varphi_{1}\right) / e$

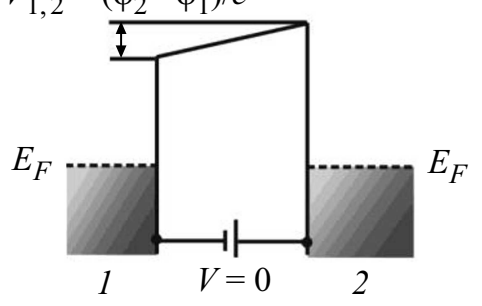

$b$

$c$
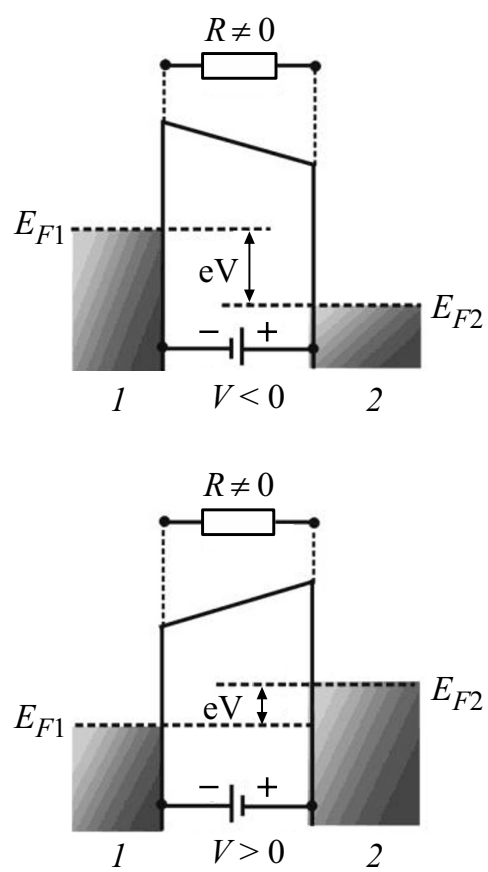

$d$

Рис. 1. Энергетические диаграммы потенциальных барьеров между двумя металлами, имеющими разные работы выхода $\varphi_{1}$ и $\varphi_{2}$.

о заполненных состояниях в объекте (спектроскопия заполненных состояний), а при $V>0$ (рис. $1, d)-$ информацию о незаполненных состояниях (спектроскопия незаполненных состояний). И те и другие зависимости, построенные в одних и тех же координатах „абсолютное значение тока - абсолютное значение напряжения смещения“ при полном подобии электродов 1 и 2, должны совпадать. Однако в действительности они вследствие ряда причин могут различаться. Эти различия могут быть обусловлены несколькими факторами. В настоящей работе предполагается, что одним из главных среди них является различие в работах выхода объекта и острия. Такое предположение основано на том, что работы выхода объекта и острия, как правило, различаются между собой, и что это различие может быть значительным, достигая величин $1 \mathrm{eV}$ и более. Такие значения сопоставимы с величинами напряжения смещения, используемыми в сканирующей туннельной спектроскопии. В этом методе регистрируемые токи при различных значениях смещения могут быть обусловлены разными механизмами. При малых напряжениях смещения и малых расстояниях между объектом и острием ток обусловлен туннелированием электронов через разделяющий электроды вакуумный барьер. В этом случае ВАХ близки к линейным, и КРП не оказывает на них влияния. При увеличении напряжения смещения и расстояния между объектом и острием все бо́льшую роль начинает играть автоэлектронная (полевая) эмиссия [7], и при больших $V$ ее вклад становится доминирующим. Это следует из того, что зависимость плотности автоэлектронного тока от работы выхода эмитирующей поверхности и напряженности электрического поля у этой поверхности для формы барьера Шоттки-Нордгейма описывается выражением [7]

$$
J=\alpha \varphi^{-1} F^{2} \exp \left[-v(f) \beta \varphi^{3 / 2} / F\right],
$$

где $\alpha$ и $\beta-$ константы, $F-$ напряженность электрического поля, $f-$ масштабный коэффициент, $v(f) \approx 1-f+(1 / 6) f \ln f$. Из (1) следует, что ток $J$ существенно зависит от работы выхода, и что, следовательно, изменение этой величины будет вызывать сдвиг характеристик, регистрируемых методом СТС, по шкале напряжения смещения. Сказанное означает, что если работы выхода объекта и острия не совпадают по величине, т. е. $V_{2.1} \neq 0$, то не будут совпадать и ВАХ для заполненных и незаполненных состояний. КРП должна, следовательно, сказываться и на производных ВАХ, каковыми являются зависимости дифференциальной проводимости $d I / d V$ от $V$. Из проведенного анализа и, в частности, из формулы (1) следует, что при указанном выше соотношении работ выхода $\varphi_{2}>\varphi_{1}$ характеристики для незаполненных состояний будут сдвигаться в область больших напряжений смещения относительно аналогичных зависимостей для заполненных состояний.

\section{2. Экспериментальная проверка выводов, вытекающих из рассмотренной модели}

Структурами, которые к настоящему моменту исследованы с помощью СТС и которые наиболее подходят для проверки выводов, вытекающих из настоящей работы, являются нанопленки иттербия толщиной 16 монослоев $(6.08 \mathrm{~nm})$, осажденные на подложки $\mathrm{Si}(111)$. Эти пленки имеют работу выхода, равную $3.3 \mathrm{eV}$ [8]. Для них в случае, когда для проведения исследований с помощью СТС используются вольфрамовые острия $(\varphi=4.5 \mathrm{eV})$, контактная разность потенциалов будет иметь значительную величину, равную $1.2 \mathrm{~V}$. Она сопоставима с величинами смещения, используемыми в методе СТС. 
В настоящем исследовании нанопленки иттербия создавались на кремниевых подложках $\mathrm{Si}(111)$. Во время осаждения иттербия температура подложек была комнатной. При указанных условиях, как было показано ранее [9], формируются атомно-резкие границы раздела $\mathrm{Yb}-\mathrm{Si}(111)$, на которых перемешивание практически отсутствует. Важной особенностью исследованных нанопленок иттербия является их высокая однородность по толщине. Так, по данным сканирующей туннельной микроскопии (СТM) при толщине 16 монослоев средняя шероховатость поверхности нанопленки составляет $4 \%$ $(0.024 \mathrm{~nm})[10]$.

Эксперименты проводились in situ с помощью описанной ранее сверхвысоковакуумной установки, в состав которой входил сканирующий туннельный микроскоп STM 1 фирмы Omicron (Германия) [10]. Основным методом, используемым в работе, была сканирующая туннельная спектроскопия. С ее помощью были получены зависимости туннельного тока I и дифференциальной проводимости $d I / d V$ от напряжения смещения $V$, прикладываемого к образцу относительно острия. Как известно [1-4], все эти зависимости содержат важные сведения об электронной структуре образцов, в частности, они отражают распределение локальной плотности состояний вблизи энергии Ферми, т.е. в области заполненных (при $V<0$ ) и незаполненных $(V>0)$ состояний. Измерения проводились при комнатной температуре. Данные СТС регистрировались одновременно с СТМ-изображениями топографии поверхности $\mathrm{Yb}-\mathrm{Si}(111)$ при начальных значениях напряжения смещения $V_{0}=1.70 \mathrm{~V}$ и туннельного тока $I_{0}=0.056 \mathrm{nA}$. Приведенные ниже зависимости получены на хорошо выраженных участках этих поверхностей, находящихся вдали от локальных дефектов. Другие детали эксперимента можно найти в работе [10].

На рис. 2 в координатах ток $I-$ абсолютное значение величины смещения $V$ приведены ВАХ, полученные методом СТС для структур $\mathrm{Yb}-\mathrm{Si}(111)$ для заполненных (кривая 1) и незаполненных (кривая 2) состояний. Кроме того, на вставке на этом рисунке более крупно показаны начальные участки BAX (малые значения напряжения смещения $|V|<0.2 \mathrm{~V})$. Видно, что эти участки практически линейны и очень близки друг к другу. При увеличении напряжения смещения ВАХ, во-первых, становятся сугубо нелинейными. А во-вторых, ВАХ для незаполненных состояний сдвигаются в область больших $|V|$ относительно аналогичных зависимостей для заполненных состояний на значительную величину. Это полностью согласуется с рассмотрением, проведенном в разд. 1. Существенно, что количественно экспериментально наблюдаемые величины сдвигов ВАХ существенно меньше значений КРП. Так, например, при значениях токов, равных $0.20,0.25,0.30$ и $0.35 \mathrm{nA}$ (на рис. 2 эти токи отмечены горизонтальными линиями), величины сдвига характеристик составляют соответственно 0.60 , $0.64,0.70$ и $0.74 \mathrm{~V}$ (непостоянство этой величины обусловлено нелинейностью зависимостей).

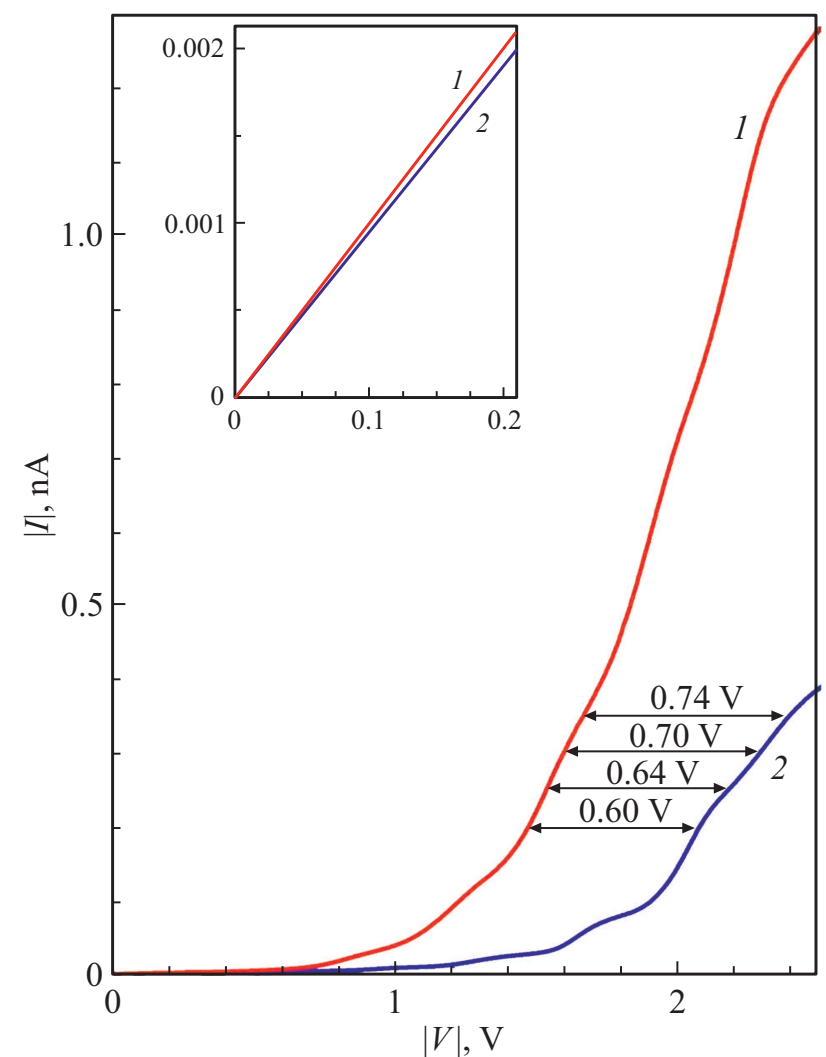

Рис. 2. ВАХ для нанопленок иттербия толщиной 16 монослоев для заполненных (1) и незаполненных (2) состояний. На вставке показана начальная область характеристик при $|V|<0.2 \mathrm{~V}$.

На рис. 3 приведены зависимости дифференциальной проводимости от напряжения смещения для заполненных (кривая 1) и незаполненных состояний (кривая 2). Основные особенности этих зависимостей повторяют аналогичные особенности результатов, приведенных на рис. 2. Однако есть и отличия. Так, на рис. 3 отчетливо просматривается целый ряд максимумов и точек перегиба. Эти особенности обусловлены квантовыми состояниями, формирующимися в нанопленках иттербия при малых их толщинах. Более подробно эти состояния рассмотрены в нашей предыдущей работе [11].

Для того чтобы понять, в каких условиях были получены экспериментальные зависимости, приведенные на рис. 2 и 3, можно воспользоваться результатами классификации ВАХ, приведенными в работе [12]. В этой работе выделены три типа ВАХ. Первый из них наблюдается при расстояниях между острием и объектом, равным $\approx 12 \AA$. При таком расстоянии ток между электродами обусловлен туннелированием электронов через вакуумный зазор. Как показал Simmons [13], BAX в этом случае практически линейны при обеих полярностях напряжений смещения и наблюдаются в области их значений, равных нескольким десятым долей вольта. 


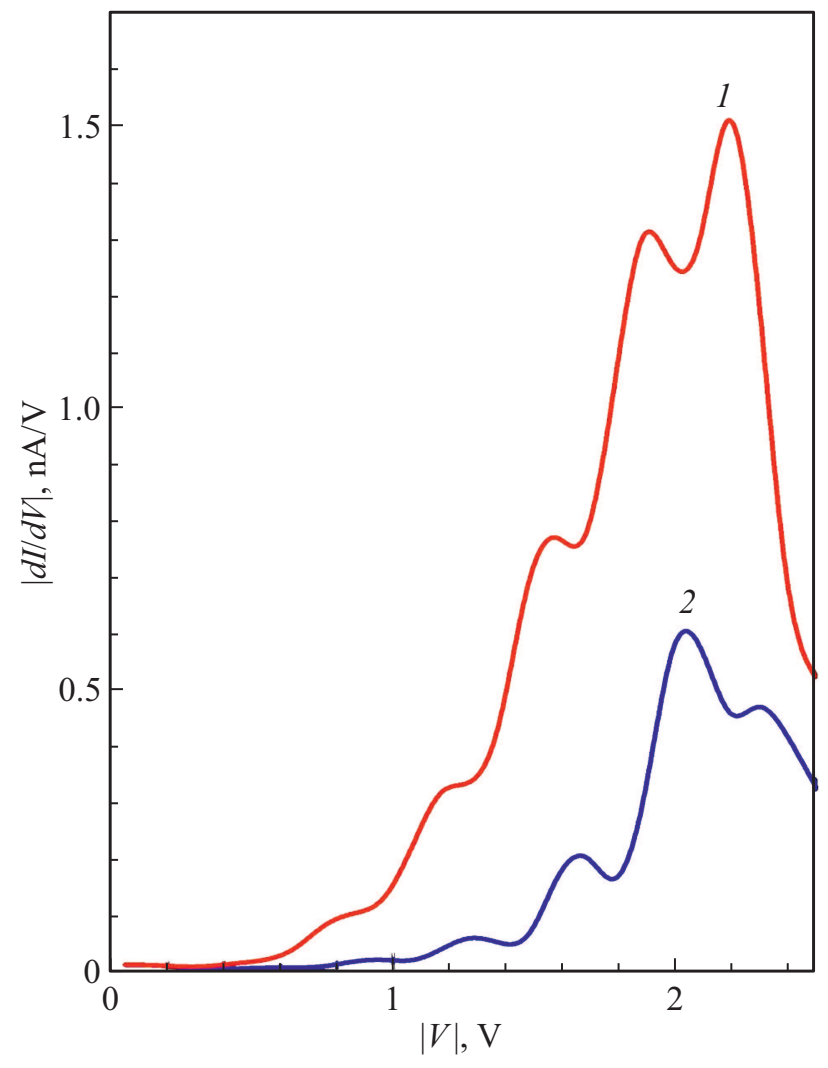

Рис. 3. Зависимости дифференциальной $(d I / d V)$ проводимости от напряжения смещения для заполненных (1) и незаполненных (2) состояний для нанопленок иттербия толщиной 16 монослоев.

Второй случай реализуется при расстояниях между острием и объектом, примерно равных $20 \AA$, и напряжениях смещения 0-3.5 V. В этом случае, который является промежуточным, ток $I$ между острием и объектом является суммой двух компонент, одна из которых определяется процессами туннелирования, а вторая обусловлена механизмом автоэлектронной (полевой) эмиссией. В этом варианте вклад этих компонент зависит от напряжения смещения. При больших значениях этого смещения вторая компонента будет доминирующей.

Наконец, в третьем случае, который реализуется при расстояниях между острием и образцом, примерно равным $27 \AA$, зависимость тока $I$ от напряжения $V$ обусловлена автоэлектронной эмиссией. Как известно, в этом режиме плотность тока эмиссии экспоненциально увеличивается с ростом напряженности электрического поля (уравнение (1)).

Согласно результатам описанного анализа, ВАХ, полученные в настоящей работе, относятся ко второму из рассмотренных трех случаев. Это означает, что одна из компонент тока $I$ обусловлена механизмом автоэлектронной (полевой) эмиссии и экспоненциально зависит от напряженности электрического поля у эмитирующей поверхности. Последнее означает, что вклад автоэлектронной эмиссии в общий ток будет возрастать при увеличении напряжения смещения. Такой вывод подтверждается экспериментальными результатами, приведенными на рис. 2 и 3. Согласно этим результатам при малых напряжениях смещения ВАХ практически линейны и совпадают для обеих полярностей напряжения $V$. При больших же напряжениях смещения зависимости нелинейны и разнесены друг относительно друга. Если предположить, что при напряжениях смещения, близких к значению $|V|=2 \mathrm{~V}$, ток обусловлен в основном механизмом полевой эмиссии, то тогда с помощью уравнения (1) можно оценить вклад изменения работы выхода при переходе от режима регистрации ВАХ для незаполненных состояний к режиму регистрации ВАХ заполненных состояний, т. е. от $\varphi_{2}=4.5 \mathrm{eV}$ к $\varphi_{1}=3.3 \mathrm{eV}$. Это можно сделать, допустив, что изменение тока $J$ в (1) обусловлено в основном изменением экспоненциального члена. Тогда для двух равных токов (при таком условии определяется сдвиг BAX друг относительно друга), но разных значениях работы выхода будет справедливо $\left(\varphi_{1} / \varphi_{2}\right)^{3 / 2}=F_{1} / F_{2}$. Так как напряженность электрического поля связана с напряжением смещения простым соотношением $F=c V$, где $c$ - постоянная, предыдущее соотношение может быть переписано в виде $\left(\varphi_{1} / \varphi_{2}\right)^{3 / 2}=V_{1} / V_{2}$. Это уравнение дает возможность рассчитать сдвиг зависимости 2 относительно зависимости 1 на рис. 2. Результаты расчетов для четырех горизонтальных сечений рис. 2 следующие: экспериментально наблюдаемым значениям сдвига 0.60 , $0.64,0.70$ и $0.74 \mathrm{~V}$ соответствуют расчетные значения $0.87,0.91,0.94$ и $0.98 \mathrm{~V}$. Видно, что расчетные значения несколько превышают экспериментально полученные величины. Это различие обусловлено, по-видимому, теми допущениями, которые были сделаны при проведении расчетов. Второй вывод, который вытекает из всех полученных в работе результатов, состоит в том, что КРП оказывает существенное влияние на ВАХ и зависимости дифференциальной проводимости от величины смещения. Наконец, сущность третьего вывода заключается в том, что величина сдвига друг относительно друга экспериментально наблюдаемых ВАХ для заполненных и незаполненных состояний существенно меньше значений КРП.

\section{Заключение}

С помощью энергетических диаграмм асимметричных потенциальных барьеров, которые формируются при контакте двух металлов, обладающих различными работами выхода, рассмотрено влияние КРП на характеристики, получаемые с помощью сканирующей туннельной спектроскопии в одних и тех же координатах ток $I$ (или производная $d I / d V)$ - абсолютное значение напряжения смещения $V$. Показано, что наличие контактной разности потенциалов должно приводить к смещению ВАХ для незаполненных состояний относительно таких же характеристик для заполненных состояний в сторону 
больших значений величин $V$. Проведено сопоставление этих выводов с полученными экспериментальными результатами. Установлено, что для нанопленок иттербия (толщина 16 монослоев), имеющих работу выхода $3.3 \mathrm{eV}$, при использовании вольфрамовых острий (работа выхода $4.5 \mathrm{eV}$ ) действительно наблюдается сдвиг ВАХ и зависимостей дифференциальной проводимости от напряжения смещения. Величина этого смещения равна 0.64-0.74 V. Она существенно меньше контактной разности потенциалов $1.2 \mathrm{~V}$ для использованной в работе пары объект (нанопленки иттербия) - вольфрамовое острие.

\section{Конфликт интересов}

Авторы заявляют, что у них нет конфликта интересов.

\section{Список литературы}

[1] G.J. Chen. Introduction to Scanning Tunneling Microscopy (University Press, Oxford 2009), 423 p.

[2] J.J. Kubby, J.J. Boland. Surf. Sci. Rep., 26, 61 (1996).

[3] H.J.W. Zandvliet, A.van Houselt. Annu. Rev. Anal. Chem., 2, 37 (2009).

[4] R.M. Feenstra, G.A. Frazier, Yu. Pan, S. Fölsch, Yu.-C. Lin, B. Jarivala, K. Zhang, A. Robinson. J. Vac. Sci. Technol. A, 39, 011001 (2021).

[5] J.G. Simmons. J. Appl. Phys., 34, 2581 (1963).

[6] J.G. Simmons. Phys. Rev. Lett., 10, 10 (1963).

[7] R.G. Forbes. Renewing the Mainstream Theory of Field and Thermal Electron Emission. Ch. 9. P. 387-447. In: G. Gaertner, W. Knapp, R.G. Forbes. Modern Developments in Vacuum Electron Sources. Topics in Applied Physics (Springer, 2020), v. 135.

[8] М.А. Митцев, М.В. Кузьмин. ФТТ, 58, 1794 (2016).

[9] Д.В. Бутурович, М.В. Кузьмин, М.В. Логинов, М.А. Митцев. ФТТ, 50,168 (2008).

[10] М.В. Кузьмин, М.А. Митцев. ЖТФ, 90 (8), 1359 (2020). DOI: $10.21883 /$ JTF.2020.08.49548.81-20

[11] М.В. Кузьмин, М.А. Митцев. ЖТФ, 91 (7), 1189 (2021). DOI: 10.21883/JTF.2021.07.50961.18-21

[12] R. Young, J. Ward, F. Sclire. Phys. Rev. Lett., 27, 922 (1971).

[13] J.G. Simmons. J. Appl. Phys., 34, 1793 (1963). 